\title{
Paraneoplastic Leukemoid Reaction in a Patient with Urothelial Carcinoma: A Case Report
}

\author{
Aleksi E. Suo ${ }^{1}$, Tahir Abbas ${ }^{2 *}$ \\ ${ }^{1}$ Department of Medicine, University of Saskatchewan, Saskatoon, Canada \\ ${ }^{2}$ Department of Oncology, Saskatchewan Cancer Agency, University of Saskatchewan, Saskatoon, Canada \\ Email: aleksi.suo@usask.ca, ${ }^{*}$ tahir.abbas@saskcancer.ca
}

Received 26 March 2014; revised 20 April 2014; accepted 3 May 2014

Copyright (C) 2014 by authors and Scientific Research Publishing Inc.

This work is licensed under the Creative Commons Attribution International License (CC BY).

http://creativecommons.org/licenses/by/4.0/

(c) (i) Open Access

\begin{abstract}
Introduction: Leukemoid reactions in cancer are rare and associated with a poor prognosis. The mechanism driving paraneoplastic leukemoid reactions appears to be gain-of-function granulocyte-colony stimulating factor (G-CSF) secretion by tumour cells. Case Presentation: A 57-year-old male smoker presented with a one-year history of painless frank hematuria and three kilograms weight loss. Cystoscopy revealed a high-grade urothelial carcinoma with sarcomatoid differentiation. The patient was treated by radical cystoprostatectomy, bilateral pelvic lymph node dissection and formation of an ileal conduit. In the absence of bone marrow infiltrations, recurrence of the urothelial carcinoma three months later was associated with a paraneoplastic leukemoid reaction with a white blood cell count peaking at $82.62 \times 10^{9} /$ l. Unfortunately, his condition continued to deteriorate and he died shortly thereafter. Conclusion: Monitoring of white blood cell counts in paraneoplastic leukemoid reactions can be a useful indicator of response of the malignancy to chemotherapy or radiotherapy and an indication of relapse after treatment. Paraneoplastic leukemoid reactions are caused by G-CSF secretion by tumour cells and are associated with a poor prognosis. Whether G-CSF signaling plays a role in the aggressive nature of these cancers is currently unknown.
\end{abstract}

\section{Keywords}

Urothelial Carcinoma, Paraneoplastic, Leukemoid Reaction

\section{Introduction}

A leukemoid reaction is defined as a persistent leukocytosis greater than $50 \times 10^{9} / 1$ [1] [2]. It can be neutrophilic "Corresponding author. 
or lymphocytic in nature and observed in a variety of clinical settings, although rarely it is seen in cancer [1] [2]. A paraneoplastic leukemoid reaction has a cancer-induced cause in the absence of leukemia and infection. The mechanism of this paraneoplastic syndrome is thought to result from tumour secretion of granulocyte-colony stimulating factor (G-CSF) [3]-[9]. Paraneoplastic leukemoid reactions have been reported in several solid-tumour types and appear to be associated with a poor prognosis.

\section{Case Presentation}

A 57-year-old male smoker with presented with a one-year history of painless frank hematuria and three kilograms weight loss. This was on a background of a 30 pack year smoking history and a baseline weight of $60 \mathrm{ki}-$ lograms just two months previous. He had a medical history significant for hepatitis $\mathrm{C}$ infection and rheumatic fever. Physical examination did not demonstrate any lymphadenopathy or hepatosplenomegaly and his blood work, including a white blood cell count, was within normal range. He underwent cystoscopy revealing a high-grade urothelial carcinoma with sarcomatoid differentiation. This was followed by a radical cystoprostatectomy, bilateral pelvic lymph node dissection and formation of an ileal conduit. His postoperative recovery was uneventful. Pathology demonstrated tumour invasion into the perivesical tissue with no lymph node involvement and computed tomography (CT) confirmed no distant metastases at that time, T3N0M0. A follow-up CT scan at three months demonstrated two new right-sided pelvic soft-tissue masses measuring $3.0 \times 2.7 \mathrm{~cm}$ and $4.0 \times 4.5 \mathrm{~cm}$. Radiological and scintigraphic studies ruled out bone metastasis. A leukocytosis was present with a peripheral white blood cell count of $41.7 \times 10^{9} / \mathrm{l}$ with $87 \%$ neutrophils, $7 \%$ lymphocytes, $5 \%$ monocytes and $1 \%$ metamyelocytes. The patient was treated with one cycle of cisplatin and gemcitabine. Chemotherapy was then withheld due to a urinary tract infection that resolved with antibiotic therapy. The patient's white blood cell count continued to increase to $74.66 \times 10^{9} / \mathrm{l}$ with $73 \%$ neutrophils, $16 \%$ bands, $2 \%$ metamyelcytes, $4 \%$ lymphocytes, $4 \%$ monocytes and $1 \%$ eosinophils with no fever or source of infection. His hemoglobin was $101 \mathrm{~g} / \mathrm{l}$, platelets were $407 \times 10^{9} /$, and peripheral smear demonstrated marked leukocytosis primarily attributable to mature neutrophils with moderate normochromic normocytic anemia. Future bone marrow studies revealed hypercellular marrow with trilineage hematopoiesis with no evidence of infiltration by tumours, myeloid or lymphoid neoplasm or myeloproliferative processes. Testing for BCR/ABL1 translocation and the JAK2 V617F and exon 12 mutations were negative. A diagnosis of paraneoplastic leukemoid reaction was established. His white blood cell count peaked at $82.62 \times 10^{9} / 1$ and a repeat CT scan revealed rapidly progressive disease affecting the liver and bowel. Unfortunately, his condition continued to deteriorate and he died 9 months after his initial diagnosis of bladder cancer and only four months from the time of cancer recurrence. An autopsy was not performed.

\section{Discussion}

The diagnostic work-up for a leukocytosis greater than $50 \times 10^{9} /$ requires the exclusion of a malignant hematological condition like chronic myelogenous leukemia (CML), chronic neutrophilic leukemia (CNL), chronic lymphocytic leukemia (CLL) and myelodysplastic / myeloproliferative disorder [1] [2]. It is also essential to rule out a bone marrow irritation and replacement by metastatic solid tumor cells. Careful examination of blood films will help in the diagnosis but definitive diagnosis rests on bone marrow samples to exclude infiltration. BCR/ ABL1 translocation and JAK2 mutation testing are usually required to discount a myeloproliferative condition [1]. Next, an underlying etiology of the leukocytosis must be identified. Commonly infection, drugs, hemorrhage or hemolysis are identified. However, on rare occasions cancer will elicit a paraneoplastic phenomenon provoking a leukemoid reaction.

Paraneoplastic leukemoid reactions have been described with different primary tumour sites including cervical, colon, skin, mesothelial, lung, penile and bladder cancers from both epithelial and mesenchymal tissue origins, although these occurrences are rare [3]-[15]. In some cases, monitoring of white blood cell counts in paraneoplastic leukemoid reactions have demonstrated to be a useful indicator of response of the malignancy to chemotherapy or radiotherapy and an indication of relapse after treatment [3] [5] [9] [13]. Interestingly, our patient did not have a leukocytosis at presentation; rather, the patient's leukemoid reaction developed after his cancer recurred.

The mechanism driving the paraneoplastic leukemoid reaction is through the production of cytokines, particularly G-CSF, inducing a leukocytosis with a left shift. Several cases of transitional cell carcinoma, and other cancer types, with leukemoid reactions have demonstrated significantly elevated levels of G-CSF [3]-[5] 
[8]-[11]. Leukemoid reactions in cancer have also been shown to bid a relatively poor prognosis. Mizutani et al. looked at 141 patients with urothelial tumours and 13 had G-CSF-related leukemoid reactions [7]. Serum G-CSF levels also correlated with a higher grade and faster progression of the cancers. Other case reports of paraneoplastic leukemoid reactions in different cancer types have also been associated with a negative prognosis, although the relationship of a rapidly progressing cancer and a leukemoid reaction has not been established [3] [5] [9] [11] [13].

It has been hypothesized that G-CSF production by tumour cells may induce autocrine signaling that accelerates growth and progression [12] [13]. G-CSF receptor expression has been demonstrated on the surface of some G-CSF secreting tumours and absent on others [3] [12]. G-CSF is also highly effective at inducing chemotaxis. Schniewind et al. postulate that G-CSF secretion may recruit WBCs to tumours and activate granulocytes to secrete proteases that assist in disintegration of surrounding tissue matrix thereby facilitating tumour growth [3].

Interestingly, G-CSF and has been shown to activate several cell signaling pathways that are common to many cancer types, for example the JAK/STAT, MAPK and PI3K/AKT pathways. Harada et al. demonstrated that G-CSF promoted survival of cardiomyocytes after myocardial infarction through JAK/STAT signaling [14]. Natori et al. demonstrated that G-CSF influenced tumour growth in murine syngeneic colon cancer cells by stimulating neovascularization [15]. It is therefore plausible for a series of gain-of-function mutations by cancer cells to induce G-CSF autocrine signaling that promote tumour growth, cancer progression and ultimately a poor prognosis. Further research may help delineate if directing targeted therapies at inhibiting G-CSF signaling in paraneoplastic leukemoid reactions may be of potential clinical benefit.

\section{Conclusion}

Although rare, paraneoplastic leukemoid reactions have been reported in several solid-tumour malignancies including bladder cancer, and appear to be associated with a poor prognosis. Monitoring of white blood cell counts in paraneoplastic leukemoid reactions can be a useful indicator of response of the malignancy to chemotherapy or radiotherapy and an indication of relapse after treatment. The mechanism driving the aggressive nature of these cancers may be related to a gain-of-function G-CSF secretion and autocrine signaling of cancer cells.

\section{Competing Interests}

The authors declare that they have no competing interests.

\section{Author's Contributions}

AS analyzed and interpreted the hematological and oncological data and was the major contributor in writing the manuscript. TA was involved in analyzing, interpreting and intellectual critique of the hematological and oncological data. All authors read and approved the final manuscript.

\section{References}

[1] George, T.I. (2012) Malignant or Benign Leukocytosis. Hematology, 2012, 475-484.

[2] Sakka, V., Tsiodras, S., Giamarellos-Bourboulis, E.J. and Giamarellou, H. (2006) An Update on the Etiology and Diagnostic Evaluation of a Leukemoid Reaction. European Journal of Internal Medicine, 17, 394-398. http://dx.doi.org/10.1016/j.ejim.2006.04.004

[3] Schniewind, B., Christgen, M., Hauschild, A., Kurdow, R., Kalthoff, H. and Klomp, H.J. (2005) Paraneoplastic Leukemoid Reaction and Rapid Progression in a Patient with Malignant Melanoma. Cancer Biology \& Therapy, 4, 23-27. http://dx.doi.org/10.4161/cbt.4.1.1447

[4] Ohbayashi, H., Nosaka, H., Hirose, K., Yamase, H., Yamaki, K. and Ito, M. (1999) Granulocyte Colony Stimulating Factor-Producing Diffuse Malignant Mesothelioma of Pleura. Internal Medicine, 38, 668-670. http://dx.doi.org/10.2169/internalmedicine.38.668

[5] Jardin, F., Vasse, M., Debled, M., Dominique, S., Courville, P., Callonnec, F., Buchonnet, G., et al. (2005) Intense Paraneoplastic Neutrophilic Leukemoid Reaction Related to a G-CSF Secreting Lung Sarcoma. American Journal of Hematology, 80, 243-245. http://dx.doi.org/10.1002/ajh.20454

[6] Ito, N., Matsuda, T., Kakehi, Y., Takeuchi, E., Takahashi, T. and Yoshida, O. (1990) Bladder Cancer Producing Gra- 
nulocyte Colony-Stimulating Factor. New England Journal of Medicine, 323, 1709-1710. http://dx.doi.org/10.1056/NEJM199012133232418

[7] Mizutani, Y., Okada, Y., Terachi, T., Kakehi, Y. and Yoshida, O. (1995) Serum Granulocyte Colony-Stimulating Factor Levels in Patients with Urinary Bladder Tumour and Various Urological Malignancies. British Journal of Urology, 76, 580-586. http://dx.doi.org/10.1111/j.1464-410X.1995.tb07782.x

[8] Nara, T., Hayakawa, A., Ikeuchi, A., Katoh, N. and Kishimoto, S. (2003) Granulocyte Colony-Stimulating Factor-Producing Cutaneous Angiosarcoma with Leukaemoid Reaction Arising on a Burn Scar. British Journal of Dermatology, 149, 1273-1275. http://dx.doi.org/10.1111/j.1365-2133.2003.05684.x

[9] Nimieri, H.S., Makoni, S.N., Madziwa, F.H. and Nemiary, D.S. (2003) Leukemoid Reaction Response to Chemotherapy and Radiotherapy in a Patient with Cervical Carcinoma. Annals of Hematology, 82, 316-317.

[10] McKee, L.C. (1985) Excess Leukocytosis (Leukemoid Reactions) Associated with Malignant Diseases. South Medical Journal, 78, 1475-1482. http://dx.doi.org/10.1097/00007611-198512000-00018

[11] Miller, J.I., Sarver, R.G. and Drach, G.W. (1994) Leukemoid Reaction: A Rare Paraneoplastic Syndrome Associated with Advanced Bladder Carcinoma. Urology, 44, 444-446. http://dx.doi.org/10.1016/S0090-4295(94)80114-2

[12] Tachibana, M. and Murai, M. (1998) G-CSF Production in Human Bladder Cancer and Its Ability to Promote Autocrine Growth: A Review. Cytokines Cellular Molecular Therapy, 4, 113-120.

[13] Dukes, J.W. and Tierney, L.M. (2009) Paraneoplastic Leukemoid Reaction as Marker for Transitional Cell Carcinoma Recurrence. Urology, 73, 17-19. http://dx.doi.org/10.1016/j.urology.2008.05.023

[14] Harada, M., Qin, Y., Takano, H., Minamino, T., Zou, Y., Toko, H., Ohtsuka, M., et al. (2005) G-CSF Prevents Cardiac Remodeling after Myocardial Infarction by Activating the Jak-Stat Pathway in Cardiomyocytes. Natural Medicine, 11, 305-311. http://dx.doi.org/10.1038/nm1199

[15] Natori, T., Sata, M., Washida, M., Hirata, Y., Nagai, R. and Makuuchi, M. (2002) G-CSF Stimulates Angiogenesis and Promotes Tumor Growth: Potential Contribution of Bone Marrow-Derived Endothelial Progenitor Cells. Biochemical and Biophysical Research Communications, 297, 1058-1061. http://dx.doi.org/10.1016/S0006-291X(02)02335-5 